Tezcan B., Bölükbaşı D., Kazancı D., Turan S., Suer Kaya G., Özgök A.

Turkiye Yuksek Ihtisas Training and Research Hospital, Dept of Anaesthesiology \& Intensive Care, Ankara, Turkey

\title{
IS SUGAMMADEX SAFE IN NEUROMUSCULAR DISEASE? THE SUCCESSFUL USE OF SUGAMMADEX IN A PATIENT WITH GUILLAIN BARRE SYNDROME
}

\begin{abstract}
Background: Sugammadex is a new neuromuscular reversal agent that is frequently used in difficult entubations and some other states like protecting the patient from side effects of anticholinesterases or for the sake of intermittant reverse of muscle relaxant agents. Life threatening emergency cases and neuromuscular diseases are both the states that security of sugammadex is unknown.In this case we reported the succesfull use of sugammadex in a patient with Guillain Barre Syndrome (GBS).
\end{abstract}

Case report: 48 years old male patient was scheduled for hemicolectomy operation with a diagnosis of colon cancer. He had lower extremity disability due to GBS for 34 years and had no any other medical problem. On the operation day; after routine premedication and monitorization in operation room; he received fentanyl, propofol and rocuronim for anesthesia induction in appropriate doses. Operation continued for 3 hours, remifentanil,sevoflurane and rocuronium were used in maintenance. At the end of the surgery sugammadex(SGX) $4 \mathrm{mg} / \mathrm{kg}$ was administered and patient was ready for extubation in four minutes and he was transferred to intensive care unit(ICU) after a successful extubation. There was no complication in the postoperative period and he was discharged after 10 days.
Discussion: GBS is an acute inflamatory polyneuropathy characterized by symetrical disability and areflexia. Patients can exhibit otonomic disfunction, malign hyprthermia, rhabdomyolisis and idiopathic reactions against to neuromuscular blocking agents and also mechanical ventilation support can be needed in postoperative period due to muscle weakness. SGX is a modified cyclodextrin and makes an inert complex with rocuronium.It is widely admitted as a safe agent in many special patient groups. Neverthless literature is not enough about the use of SGX in neuromuscular diseases. Since it reverses the neuromuscular block more rapid and effective way when compared to anticholinesterases, SGX may be advantageous for the reversal of neuromuscular blockage especially in patients with neuromuscular disorders since they may cause elongated neuromuscular block. In our case SGX cause no complication in the perioperative period and progressed a succesfull anaesthetic management.

Learning points: The efficacy and safety of SGX in patients with neuromuscular disorders have not been determined in the literature. In our case SGX is successfully used for reversing neuromuscular blockage in a patient with GBS. 